\title{
Effective excitation by single magnet in rotation sensor and domain wall displacement of $\mathrm{FeCoV}$ wire
}

\author{
Takashi Kohara, ${ }^{1, a)}$ Tsutomu Yamada, ${ }^{1}$ Susumu Abe, ${ }^{2}$ Shiro Kohno, ${ }^{3}$ Fumio Kaneko, ${ }^{3}$ \\ and Yasushi Takemura ${ }^{1}$ \\ 'Department of Electrical and Computer Engineering, Yokohama National University, 79-5 Tokiwadai, \\ Hodogaya-ku, Yokohama 240-8501, Japan \\ ${ }^{2}$ Department of Electronics and Informatics Frontiers, Kanagawa University, 3-27-1 Rokkakubashi \\ Kanagawa-ku, Yokohama 221-8686, Japan \\ ${ }^{3}$ Nikkoshi Co., Ltd, 1-6-1 Nihonbashi honcho, Chuo-ku, Tokyo 103-0023, Japan
}

(Presented 17 November 2010; received 17 September 2010; accepted 17 December 2010; published online 6 April 2011)

\begin{abstract}
Recently, a sensor structure consisting of magnetic wires attached directly to a rotating shaft, a pick-up coil, and a pair of magnets separately located outside the rotor was proposed. This paper presents a structure whose excitation source is just a single magnet. This structure decreases the cost of the previous sensor while retaining its advantages. For example, multiple output signals can be obtained per turn of the shaft by simply adding a corresponding number of wires, without requiring more pick-up coils and magnet pairs. In this study, the dependence of the output voltage on the pick-up coil was measured and the domain wall structure was elucidated by the measurement result. (C) 2011 American Institute of Physics. [doi:10.1063/1.3564948]
\end{abstract}

\section{INTRODUCTION}

The reversal of magnetization in magnetic wires with bistable magnetization states induces a pulse voltage in a nearby pick-up coil. ${ }^{1,2}$ The amplitude of the voltage does not depend on the frequency of the applied magnetic field down to zero. The reversal of magnetization is accompanied by a large Barkhausen jump. This phenomenon has been exploited in various designs for rotation and speed and in other sensors that use a twisted $\mathrm{FeCoV}$ wire; $\mathrm{FeCoV}$ wire is considered the optimum material for this purpose because it exhibits a phenomenon known as the Wiegand effect. ${ }^{3}$ That is, the wire exhibits uniaxial magnetic anisotropy along its length. Its coercive force is about $20 \mathrm{Oe}$ in the outer shell (soft layer) and $80 \mathrm{Oe}$ in the inner core (hard core). ${ }^{4}$

In previous designs, magnetization reversal was directly detected by winding a pick-up coil around the wire, which was excited by a pair of permanent magnets attached to the rotating shaft. In order to obtain multiple pulses of output voltage in the pick-up coil during one turn of the shaft, the number of magnet pairs or wire/pick-up coil sets must be increased. However, a change of the magnetic flux leakage caused by the magnetization reversal of the $\mathrm{FeCoV}$ wire was observed. A sensor configuration that uses a pick-up coil outside the wire has therefore been proposed. ${ }^{5}$ This structure offers several advantages. For example, multiple output signals can be obtained per turn of the shaft by simply adding a corresponding number of wires, without requiring more pick-up coils and magnet pairs.

A more recent structure also has been proposed. The previous sensor structure required a pair of exciting magnets. However, the latest proposed sensor is constructed using a single magnet as the source of excitation. This sensor retains the advantages of the previous one and has a reduced cost,

${ }^{a)}$ Electronic mail: d09gd134@ynu.ac.jp. because it uses a single magnet. In this paper, we first provide details of the proposed structure. Next, we describe the measurement of the dependence of the output voltage on the pick-up coil and derivation of the domain wall motion driven by a single magnet from the measurement result.

\section{SENSOR STRUCTURE}

First, the conventional structure is explained. Figure 1(a-1) shows the structure of the combined wire and pick-up coil in which the pick-up coil is wound around the wire. Some pairs of magnets with opposite magnetic charges are applied to the alternating magnetic field. In the pick-up coil, the induced electromotive force is accompanied by a rapid magnetization reversal in the soft layer, as shown in Fig. 1(a-2).

Second, Fig. 1(b-1) shows the proposed structure in which the wire and pick-up coil are separate. The wire is attached to a rotating shaft. The single magnet is setup around the shaft such that the magnetization direction is at a right angle to the wire. When the shaft is rotating, half of the wire is exposed to an alternating magnetic field along the length of the wire and the other half is exposed to a reverse magnetic field generated from the single magnet, as shown in Fig. 2. Then, the magnetic field induces a rapid magnetization reversal in the soft layer of the wire, and the leakage of magnetic flux external to the wire changes, as shown in Fig. 1(b-2). As a result, an electromotive force induced by the magnetization reversal appears in a planar coil around the wire.

\section{EXPERIMENT}

The proposed sensor detects the change in the magnetic flux leakage caused by a pick-up coil. Therefore, the output characteristics reveal the distribution of magnetic flux leakage and the magnetic domain structure. The characteristics were measured with changes in the location and size of the pick-up coil. 

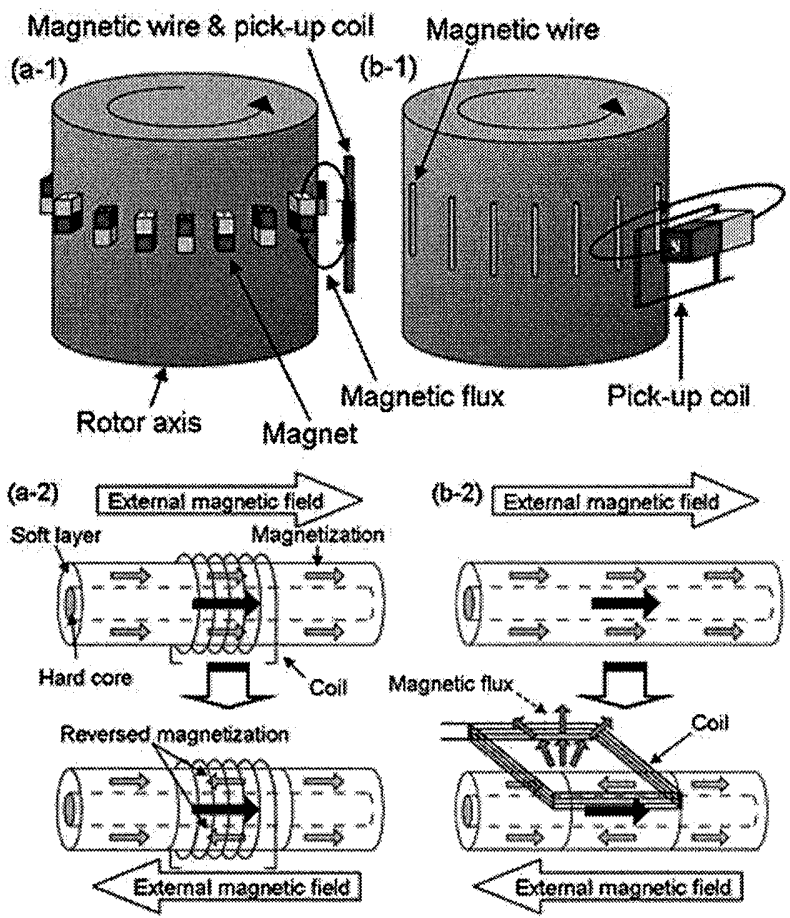

FIG. 1. Configurations of magnetic wire, magnets, and pick-up coil for rotation sensor in (a) conventional and (b) proposed methods, and detection of magnetization reversal of magnetic wire by (a) conventional and (b) proposed methods.

A twisted $\mathrm{FeCoV}$ wire with a diameter of $0.25 \mathrm{~mm}$ and length of $20 \mathrm{~mm}$, a $3 \mathrm{~mm} \times 3 \mathrm{~mm} \mathrm{NdFeB}$ magnet with a length of $5 \mathrm{~mm}$ (and a magnetization direction of $5 \mathrm{~mm}$ ), a planar rectangle-type coil, and a 35-mm-radius acrylic pipe that served as a rotor axis were used.

\section{RESULTS AND DISCUSSION}

\section{A. Alignment of pick-up coil}

First, in order to elucidate the domain wall structure at magnetization reversal, the output characteristics were meas- ured after changing the location of the pick-up coil. The pick-up coil was $2 \mathrm{~mm} \times 10 \mathrm{~mm}$ and had 100 turns. The single magnet was placed $4 \mathrm{~mm}$ from the rotating shaft and the wire was placed $2 \mathrm{~mm}$ from the pick-up coil, as shown in Fig. 3(a). The output voltage was measured for various positions of pick-up coil to along the length of the wire. As shown in Fig. 3(b), when the output voltages peaked, the maximum value of the magnetic flux leakage from the domain wall was detected by the pick-up coil. Therefore, the position of the domain wall on the wire can be estimated.

Second, in order to observe the magnetization of the $\mathrm{FeCoV}$ wire at magnetization reversal, the pick-up coil was wound directly around the wire, and after changing the location of the pick-up coil, the output voltage was measured for each alignment, as shown in Fig. 4(a). The pick-up coil was $2 \mathrm{~mm}$ wide and had 100 turns. Output voltages were induced in the pick-up coil both before and after the wire passed in front of the excited magnet, as shown in Fig. 4(b). This is because magnetization reversal is caused and the inverse magnetic field is applied after the wire moves, as shown in Fig. 4(b). The output characteristics are shown in Figs. 4(c)-before-and 4(d)-after. In this experiment, magnetization reversal occurred at the place where output voltages were induced in the pick-up coil. Therefore, the domain structure of the wire was estimated from the result, as shown in Figs. 4(c-2) and (d-2). Further, it was observed that the larger the magnitude of the applied magnetic field, the greater the induced output voltage in the pick-up coil, as shown in Figs. 4(c-2) and (d-2). This is because the range of magnetization reversal is enlarged by a large magnetic field and the magnetic flux leakage as the domain wall grows larger. A comparison of Figs. 4(c-2) and 4(d-2) shows that the output voltage in the former is always more than in the latter case. This is because the effects of the demagnetizing field and the magnetostatic coupling in the wire are different before and after the wire passes in front of the excited magnet. ${ }^{6}$

\section{B. Domain structure}

Comparison of the domain structures in Figs. 3 and 4 reveals that the edges of the domain structure are different. In (a)

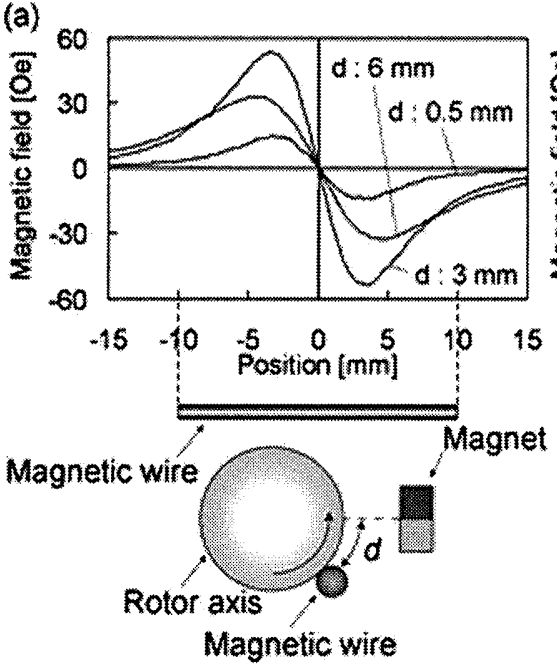

(b)

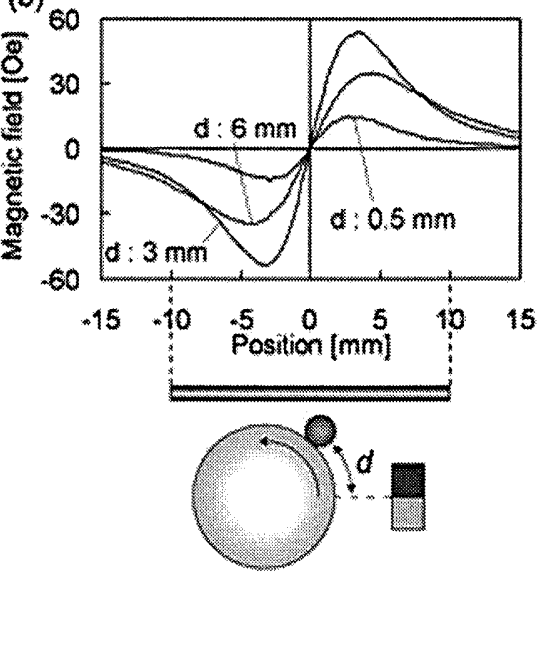

FIG. 2. Magnitude of applied magnetic field on wire (a) before and (b) after the wire passes in front of the excited magnet. 
(a)

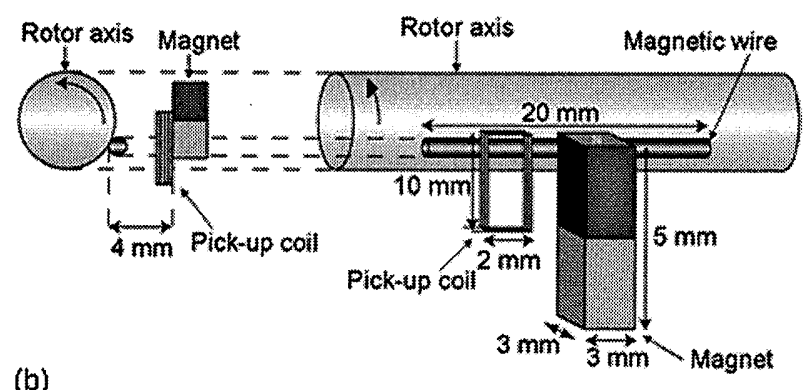

(b)

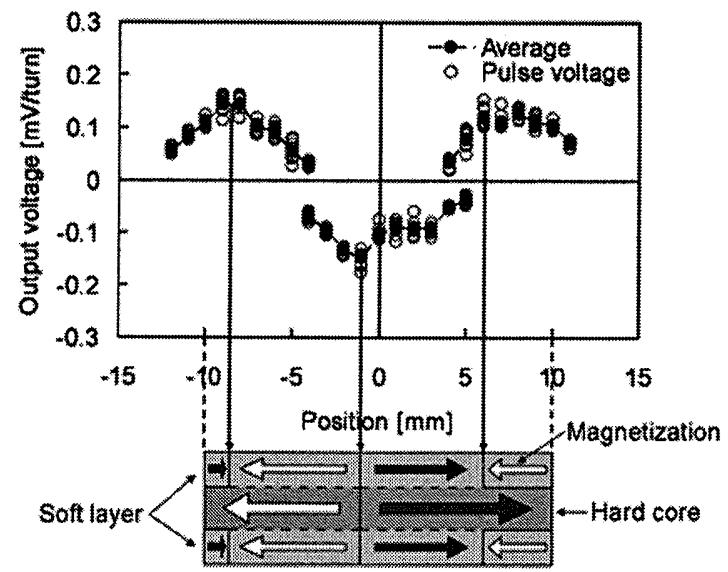

FIG. 3. Dependence of output voltage induced by twisted FeCoV wire on horizontal location of pick-up coil: (a) experimental setup, and (b) output voltage and domain structure of wire.

the case shown in Fig. 3, the position of the domain wall was determined by detecting the magnetic flux leakage of the FeCoV wire. On the other hand, in the case shown in Fig. 4, a change in magnetization was observed as the pick-up coil was wound directly around the wire. However, there is a possibility that the magnetic flux leakage is detected by the wound pick-up coil at the same time. Therefore, the domain wall on the edge of the wire was not able to be verified in Fig. 4. In addition, when the measurement was carried out after the soft layer and hard core of the $\mathrm{FeCoV}$ wire were magnetized sufficiently in the same direction, the output voltage was not induced. This is because the reverse magnetic domain, which is the source of magnetization reversal, does not exist at the edge of the wire. Therefore, the domain structure at magnetization reversal resembles that shown in Fig. 3.

\section{CONCLUSION}

This paper sets forth a rotation sensor structure in which a single magnet is used and the FeCoV wire and pick-up coil are separate from each other. The advantage of this change in structure is that it offers greater flexibility in the design of (a)

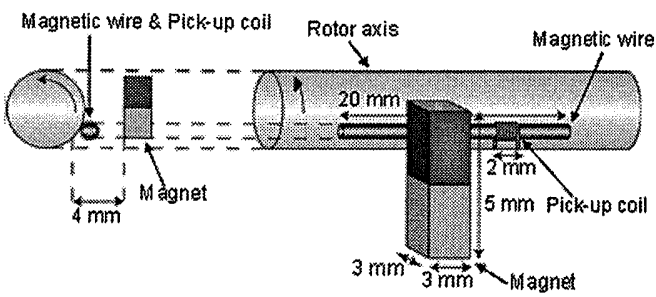

(b)

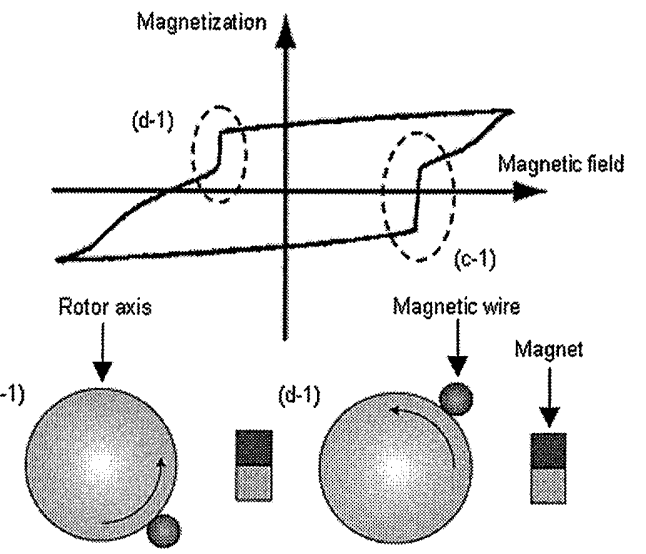

$(c-2)$

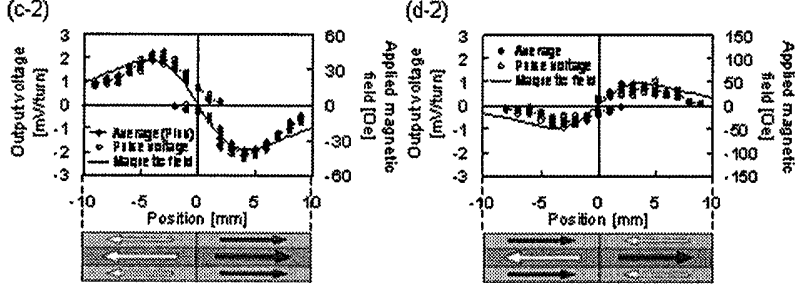

FIG. 4. Dependence of output voltage induced by twisted FeCoV wire on horizontal location of pick-up coil wound around wire: (a) experimental setup, (b) hysteresis loop of magnetic wire, and (c) before and (d) after the wire passes in front of the excitation magnet.

a motor vehicle axle, because a single excitation magnet does not need to be attached to the rotating shaft. Further, by simply increasing the number of wires on the rotating shaft, an equal number of pulse voltages are obtained. Finally, the domain structure at magnetization reversal could be estimated by measuring the output characteristics for different locations and sizes of the pick-up coil.

${ }^{1}$ R. Malmhall, K. Mohri, F. B. Humphrey, T. Manabe, H. Kawamura, J. Yamasaki, and I. Ogasawara, IEEE Trans. Magn. 23, 3242 (1987).

${ }^{2} \mathrm{M}$. Vázquez, C. Gómez-Polo, D.-X. Chen, and A. Hernando, IEEE Trans. Magn. 30, 907 (1994).

${ }^{3}$ J. R. Wiegand and M. Velinsky, U.S. Patent 3,820,090, (25 June 1974),

${ }^{4}$ S. Abe, A. Matsushita, and M. Naoe, IEEE Trans. Magn. 33, 3916 (1997).

${ }^{5}$ T. Kohara, T. Kusunoki, T. Yamada, T. Suzuki, H. Fujimoto, Y. Takemura,

S. Abe, S. Kohno, H. Itoi and F. Kaneko, 11th Joint MMM/Intermag Conference, Washington, DC, 18-22 January 2010, FW-06.

${ }^{6} \mathrm{Y}$. Takemura, T. Aoki, H. Tanaka, T. Yamada, S. Abe, S. Kohno, and H. Nakamura, IEEE Trans. Magn. 42, 3300 (2006). 